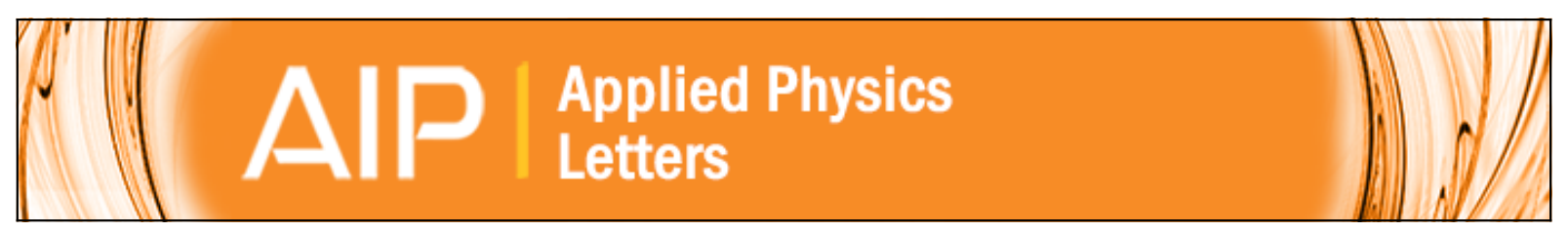

\title{
Time resolved magneto-optical studies of ferromagnetic InMnSb films
}

M. Frazier, R. N. Kini, K. Nontapot, G. A. Khodaparast, T. Wojtowicz, X. Liu, and J. K. Furdyna

Citation: Applied Physics Letters 92, 061911 (2008); doi: 10.1063/1.2842423

View online: http://dx.doi.org/10.1063/1.2842423

View Table of Contents: http://scitation.aip.org/content/aip/journal/apl/92/6?ver=pdfcov

Published by the AIP Publishing

\section{Over 700 papers \& presentations on multiphysics simulation visw now}

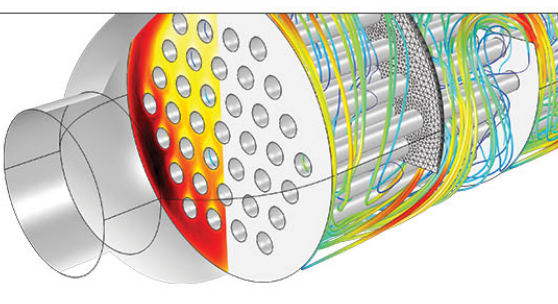




\title{
Time resolved magneto-optical studies of ferromagnetic InMnSb films
}

\author{
M. Frazier, ${ }^{1}$ R. N. Kini, ${ }^{1, a)}$ K. Nontapot, ${ }^{1}$ G. A. Khodaparast,,$\left.{ }^{1, b}\right)$ T. Wojtowicz, ${ }^{2}$ X. Liu, ${ }^{3}$ and \\ J. K. Furdyna ${ }^{3}$ \\ ${ }^{1}$ Department of Physics, Virginia Tech, Blacksburg, Virginia 24061, USA \\ ${ }^{2}$ Institute of Physics, Polish Academy of Sciences 02-668 Warsaw Poland \\ ${ }^{3}$ Department of Physics, University of Notre Dame, Notre Dame, Indiana 46556, USA
}

(Received 13 October 2007; accepted 18 January 2008; published online 15 February 2008)

\begin{abstract}
We report time resolved magneto-optical measurements in InMnSb ferromagnetic films with $2 \%$ and $2.8 \%$ Mn contents grown by low temperature molecular beam epitaxy. In order to probe a possible interaction between the spins of photoexcited carriers and the Mn ions, we measured spin dynamics before and after aligning the $\mathrm{Mn}$ ions by applying an external magnetic field at temperatures above and below the samples' Curie temperatures. We observed no significant temperature or magnetic field dependence in the relaxation times and attribute the observed dynamics entirely to the relaxation of photoexcited electrons in the conduction band where the $s-d$ coupling with the localized Mn ions is significantly weaker compared to the $p$ - $d$ exchange coupling. We observed several differences in the optical response of our InMnSb samples which could have been influenced mainly by the samples' growth conditions. () 2008 American Institute of Physics.
\end{abstract} [DOI: $10.1063 / 1.2842423$ ]

In III-Mn-V ferromagnetic semiconductors, it is known that the $s$ - $d$ coupling with the localized Mn ions is significantly weaker compared to the $p$ - $d$ exchange coupling characterizing the valence band. The sensitivity of magnetic order to the hole density due to strong $p$ - $d$ exchange coupling between holes and the embedded localized magnetic moments has been demonstrated experimentally. ${ }^{1-5}$ This effect has important consequences for developing spin based devices and makes InMnSb alloy with much higher hole mobility than the other III-Mn-V ferromagnetic semiconductors, more attractive for possible device applications. Recently Andreev reflection measurements suggest up to $52 \%$ spin polarization in ferromagnetic InMnSb, thus this material maybe of special interest as a source of spin-polarized carriers. ${ }^{6}$

Our earlier measurements ${ }^{7}$ demonstrated modifications of the relaxation times as a function of photoexcited carrier density, which can be explained using the Elliot ${ }^{8}$-Yefet relaxation mechanism. Also, the earlier carrier/spin relaxations measurements did not show any temperature dependence above and below the samples' $T_{C}$ suggesting that photoinduced carriers/spins may not be interacting strongly with the $\mathrm{Mn}$ ions. To further explore this fact, in this work, we have carried out time-resolved measurements of the relaxations before and after aligning the $\mathrm{Mn}$ ions by an external magnetic field, at temperatures above and below the samples $T_{C}$. Our second motivation to pursue these magneto-optical measurements was the recent ultrafast measurements of InMnAs ferromagnetic semiconductor. In the work on InMnAs, spin dynamics indicated fast, less than $1 \mathrm{ps}$, as well slow relaxation components $\sim 100 \mathrm{ps.}{ }^{9}$ The fast component has been interpreted as spin heating $s p$ - $d$ exchange interaction between photoexcited carrier and $\mathrm{Mn}$ ions and the slow component as spin-lattice interaction. The examples of our

\footnotetext{
${ }^{a)}$ Present address: National Renewable Energy Laboratory, Golden, CO 80401

${ }^{\text {b) }}$ Author to whom correspondence should be addressed. Electronic mail: khoda@vt.edu.
}

magneto-optical measurements at different temperatures, magnetic field strength, and excitation wavelengths are presented below.

The carrier and spin relaxation measurements were carried out using degenerate pump-probe and magneto-optic Kerr effect (MOKE) techniques. Both the pump and probe were pulses from a Ti:sapphire laser with an adjustable wavelength from 750 to $850 \mathrm{~nm}$. Each pulse had a duration of $100 \mathrm{fs}$, with a repetition rate of $80 \mathrm{MHz}$. The pump/probe power ratio was 10:1, with a maximum average pump power of around $150 \mathrm{~mW}$ on the samples. The samples were mounted inside a $10 \mathrm{~T}$ superconducting magnet with the $Z$-cut quartz windows. The pump was circularly polarized with a quarter-wave plate to generate spin-polarized carriers; due to the interband selection rules, circularly polarized light will excite carriers preferentially with one spin polarization. The probe was rotated by a half-wave plate to obtain equal $s$ and $p$ components; the reflected probe signal was then split into components with a Wollaston prism. The Kerr rotation was measured using a balanced detector and a lock-in amplifier; the magnetization generated by the spin-polarized carriers causes a Kerr rotation which manifests in a difference between the $s$ and $p$ components of the probe signal. In our measurements, the photoinduced carrier density was in the order of $\sim 10^{17} \mathrm{~cm}^{-3}$, less than the background hole density.

The measurements were performed on three InMnSb samples grown by low temperature molecular beam epitaxy with $T_{C} \sim 10 \mathrm{~K}$. The samples were grown on a GaAs substrate with CdTe layer of $\sim 4.5 \mu \mathrm{m}$, a buffer layer of InSb $(0.1 \mu \mathrm{m})$, and a $0.23 \mu \mathrm{m}$ layer of InMnSb. The samples and their characteristics are listed in Table I. In addition to different $T_{\mathrm{Mn}}$, resulting in nominally different $\mathrm{Mn}$ fluxes, the samples during the growth might as well had slightly different substrate temperature, and $\mathrm{Sb}$, and In fluxes. Since properties of III-Mn-V are to a large extent predetermined by the number of compensating defects, such as Mn interstitials, which, in turn, depend crucially on the growth conditions, the samples grown even at nominally the same conditions but in different days, might not be identical. ${ }^{10-13}$ 
TABLE I. List of the InMnSb samples studied. All samples have $0.23 \mu \mathrm{m}$ of active layer and are $p$ type. $T_{\mathrm{Mn}}$ is the Mn effusion's cell temperature, which resulted in different Hall resistivities and hysteresis loops.

\begin{tabular}{ccccc}
\hline \hline Sample & $\begin{array}{c}\text { Density } \\
\left(\mathrm{cm}^{-3}\right)\end{array}$ & $\begin{array}{c}\text { Mobility } \\
\left(\mathrm{cm}^{2} / \mathrm{V} \mathrm{s}\right)\end{array}$ & $\begin{array}{c}T_{\mathrm{Mn}} \\
\left({ }^{\circ} \mathrm{C}\right)\end{array}$ & $\begin{array}{c}\text { Mn content } \\
(\%)\end{array}$ \\
\hline $\mathrm{InMnSb}(\mathrm{A})$ & $2 \times 10^{20}$ & 100 & 690 & 2.0 \\
$\mathrm{InMnSb}(\mathrm{B})$ & $2 \times 10^{20}$ & 100 & 700 & 2.0 \\
$\mathrm{InMnSb}(\mathrm{D})$ & $2 \times 10^{20}$ & 100 & 720 & 2.8 \\
\hline \hline
\end{tabular}

Figure 1(a) demonstrates the MOKE signal for sample D (with $2.8 \% \mathrm{Mn}$ ) compared to the one observed in sample A (with 2\% Mn), and Fig. 1(b) shows the temperature dependence of the MOKE signal for sample D. The observed signals for samples $\mathrm{A}$ and $\mathrm{D}$ show relaxation times of 1 and $1.8 \mathrm{ps}$, almost a factor of 10 faster compared to our earlier reported spin relaxation times of 10 and 25 ps for samples B and $\mathrm{C}$, respectively. ${ }^{7}$ We believe that the differences are influenced by the samples' individual characteristics as a result of the slight variation in their growth conditions. The data presented in Fig. 1(b) for sample D (with $2.8 \% \mathrm{Mn}$ ) show no strong temperature dependence below and above the $T_{C}$, similar to our earlier measurements on the samples with $2.0 \% \mathrm{Mn},{ }^{7}$ suggesting possible lack of interaction between the spins of photoexcited carriers and the Mn ions.

In order to really demonstrate the insensitivity of photoexited carriers to the magnetic ordering, we performed timeresolved magneto-optical measurements using a superconducting magnet with optical access. We measured the spin relaxation at zero field and after applying a field. Earlier transport measurements on these structures demonstrated the saturation field of $0.04 \mathrm{~T},{ }^{10,11}$ and as shown for sample A in Fig. 2, our typical applied field was larger $(0.7 \mathrm{~T}$ in this case). The field was set back to zero during the measurements. Figure 2(a) shows an example of the measurements on sample A with $2 \% \mathrm{Mn}$ concentration. The absence of any strong modification in the MOKE signal after aligning the $\mathrm{Mn}$ ions implies lack of strong interaction between the ions and the spin of the photoexcited carriers via $s p-d$ exchange interaction unlike to that observed in InMnAs.'

In addition to the spin-dynamics features, we observed differences in the photoinduced carrier relaxations and the results are presented as follows. Figure 3(a) demonstrates the
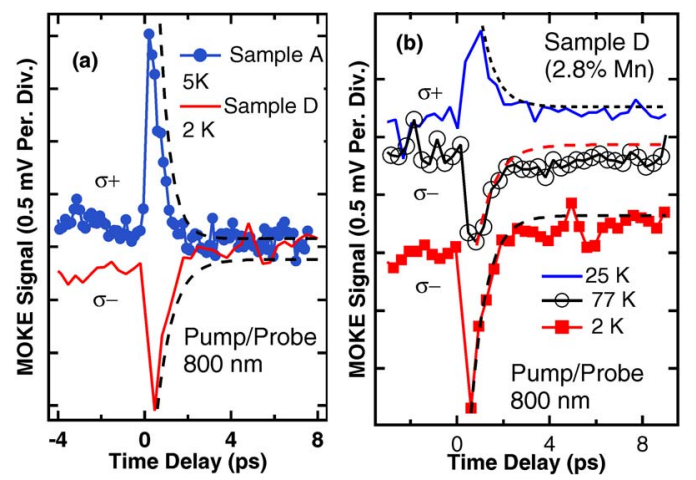

FIG. 1. (Color online) (a) MOKE signals represent spin relaxation times of samples $\mathrm{A}$ and $\mathrm{D}$ below the $T_{C}$. For clarity, the traces are plotted for two different pump circular polarizations. (b) MOKE signal of sample D with $2.8 \% \mathrm{Mn}$ content at different temperatures not showing significant temperature dependence. The dashed lines are single exponential fits to the data and are shifted slightly for clarity.

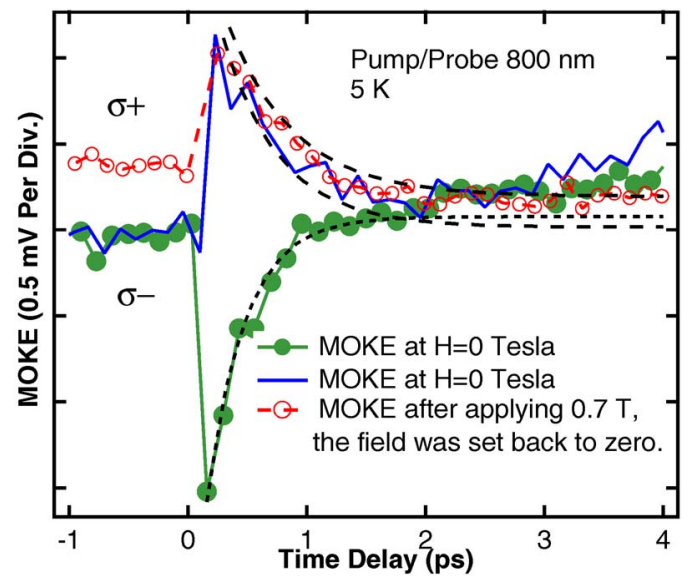

FIG. 2. (Color online) Spin relaxation of sample A for temperatures below $T_{C}$ at zero field for two circular polarizations of light and after applying $0.7 \mathrm{~T}$ of applied field. The field was set back to zero during the measurements. The dashed lines are single exponential fits to the data and are shifted slightly for clarity.

carrier relaxations at several near infrared (NIR) pump/probe wavelengths. At $800 \mathrm{~nm}$, the initial recovery of the signal occurs at $\sim 1.3 \mathrm{ps}$ compared to $\sim 3.7 \mathrm{ps}$ for the other two wavelengths. One would expect such a wavelength dependence in GaMnAs where both band filling and induced absorption can contribute in the NIR range to the relaxation dynamic; $;{ }^{14}$ this fails to explain our observations of wavelength dependence; the pump and probe energies used are far larger than the bandgap for InMnSb. In addition, the trapped electron states are considered to be formed in the midgap where the reabsorption must be insensitive for probe energy ranges used in this work. A similar wavelength dependence, as shown in Fig. 3(a), was observed in sample A but not in sample D with $2.8 \% \mathrm{Mn}$ content, where the trapped states expect to exist as well. As shown in Fig. 3(b), we were not expecting the photoinduced carriers to interact strongly with the aligned $\mathrm{Mn}$ ions after applying an external field above and below the $T_{C}$. We see nearly identical traces, indicating no dependence on the temperature or applied field. Similarly, as shown in Fig. 4(a) for sample D (with $2.8 \% \mathrm{Mn}$ ), the temperature dependence of photoinduced carrier density is not significant, similar to our earlier observations on InMnSb
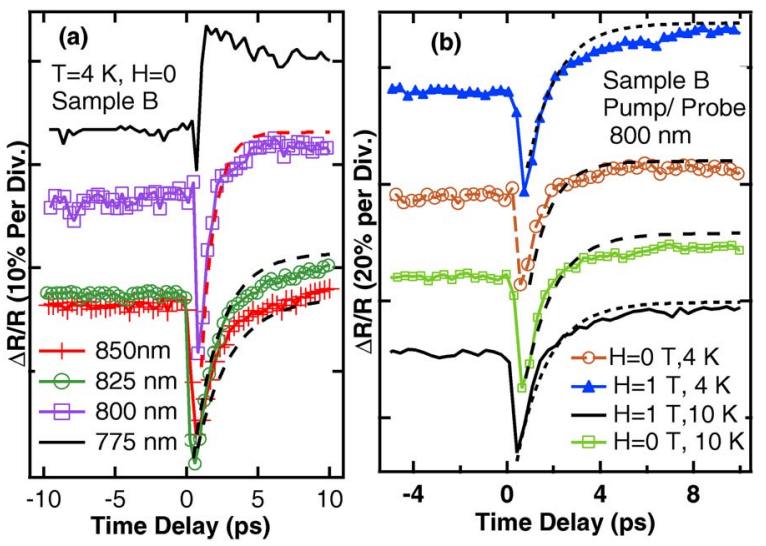

FIG. 3. (Color online) (a) Wavelength dependence of differential reflectivity of sample B for temperatures around and below $T_{C}$. (b) Carrier relaxation of sample B at temperatures below and around $T_{C}$ at $0 \mathrm{~T}$ and after applying $1 \mathrm{~T}$ (the data only for 4 and $10 \mathrm{~K}$ are plotted). The dashed lines are single exponential fits to the data and are shifted slightly for clarity. 

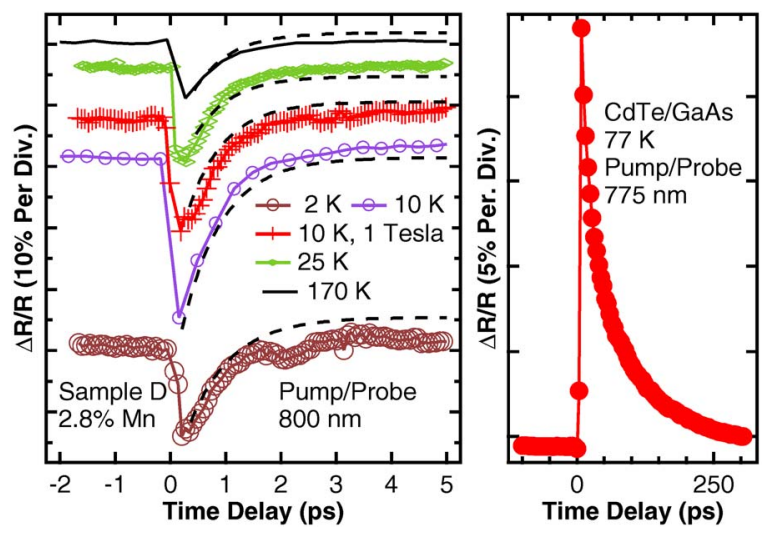

FIG. 4. (Color online) (a) Carrier relaxation of sample D for temperatures around and below $T_{C}$. The effect of external magnetic field on the relaxation was tested and is plotted for the data at $10 \mathrm{~K}$. The dashed lines are single exponential fits to the data and are shifted slightly for clarity. (b) Differential reflectivity signal from $\mathrm{CdTe} / \mathrm{GaAs}$ template sample.

ferromagnetic films with $2.0 \%$ Mn concentration. ${ }^{7}$ Lack of temperature and field dependence has also been reported previously in GaMnAs containing $2 \% \mathrm{Mn}^{15}$

In order to probe the possible contribution from the $\mathrm{CdTe} / \mathrm{GaAs}$ layers to our measurements presented, temperature and wavelength dependences of the photoinduced carrier relaxations were measured on a template with $\mathrm{CdTe}$ $\sim 4.5 \mu \mathrm{m}$. No differential reflectivity signal was observed from the template sample in the temperature range of our measurements on the InMnSb films. In addition, as shown in Fig. 4(b) when a signal was detected, the relaxation in the $\mathrm{CdTe} / \mathrm{GaAs}$ is significantly different compared to the ferromagnetic films. Therefore, neither the picture of trapped states, which can explain the wavelength dependence in GaMnAs, ${ }^{14}$ nor the contribution from the substrate can explain the wavelength dependence observed in samples $\mathrm{A}$ and $\mathrm{B}$.

In summary, we measured the magneto-optical response of optically excited spins and carriers in a series of ferromagnetic InMnSb to probe the possible contribution of $\mathrm{Mn}$ ions to the relaxation dynamics which has been reported in InMnAs (Ref. 9 with a larger remanent magnetization compared to InMnSb. The observed carrier/spin dynamics in our InMnSb were found to be influenced by the samples' growth conditions, rather than by the Mn concentrations. We observed no significant temperature or field dependence in the relaxation times supporting our earlier argument ${ }^{7}$ to attribute the observed photoinduced carrier/spin dynamics entirely to the relaxation of photoexcited electrons in the conduction band where the $s-d$ coupling with the localized Mn ions is significantly weaker compared to the $p-d$ exchange coupling. This effect can have important consequences for applications of narrow gap ferromagnetic semiconductors such as InMnSb in developing spin based devices, since these materials have much higher hole mobility than the other III-Mn-V ferromagnetic semiconductors.

This work has been supported by NSF (DMR-0507866), AFOSR Young Investigator Program (06NE231), Jeffress Trust Fund J748, and NSF (DMR06-03752).

${ }^{1}$ H. Ohno, D. Chiba, F. Matsukura, T. Omiya, E. Abe, T. Dietl, Y. Ohno, and K. Ohtani, Nature (London) 408, 944 (2000).

${ }^{2}$ S. Koshihara, A. Oiwa, M. Hirasawa, S. Katsumoto, Y. Iye, C. Urano, H. Takagi, and H. Munekata, Phys. Rev. Lett. 78, 4617 (1997).

${ }^{3}$ A. Oiwa, T. Slupinski, and H. Munekata, Appl. Phys. Lett. 78, 518 (2001).

${ }^{4}$ A. Oiwa, Y. Mitsumori, R. Moriya, T. Slupinski, and H. Munekata, Phys. Rev. Lett. 88, 137202 (2002).

${ }^{5}$ K. C. Ku, S. J. Potashnik, R. F. Wang, S. H. Chun, P. Schiffer, and N. Samarth, M. J. Seong, A. Mascarenhas, E. Johnston-Halperin, R. C. Myers, A. C. Gossard, and D. D. Awschalom, Appl. Phys. Lett. 82, 2302 (2003).

${ }^{6}$ R. P. Panguluri, K. C. Ku, T. Wojtowicz, X. Liu, J. K. Furdyna, Y. B. Lyanda-Geller, N. Samarth, and B. Nadgorny, Phys. Rev. B 72, 054510 (2005).

${ }^{7}$ K. Nontapot, R. N. Kini, A. Gifford, T. R. Merritt, G. A. Khodaparast, T. Wojtowicz, X. Liu, and J. K. Furdyna, Appl. Phys. Lett. 90, 143109 (2007).

${ }^{8}$ R. J. Elliot, Phys. Rev. 96, 266 (1954).

${ }^{9}$ J. Wang, C. Sun, Y. Hashimoto, J. Kono, G. A. Khodaparast, L. Cywinski, L. J. Sham, G. D. Sanders, C. J. Stanton, and H. Munekata, J. Phys.: Condens. Matter 18, R501 (2006).

${ }^{10}$ T. Wojtowicz, G. Cywinski, W. L. Lim, X. Liu, M. Dobrowolska, J. K. Furdyna, K. M. Yu, W. Walukiewicz, G. B. Kim, M. Cheon, X. Chen, S. M. Wang, and H. Luo, Appl. Phys. Lett. 82, 4310 (2003).

${ }^{11}$ T. Wojtowicz, W. L. Lim, X. Liu, G. Cywinski, M. Kutrowski, L. V. Titova, K. Yee, M. Dobrowolska, J. K. Furdyna, K. M. Yu, W. Walukiewicz, G. B. Kim, M. Cheon, X. Chen, S. M. Wang, H. Luo, I. Vurgaftman, and J. R. Meyer, Physica E (Amsterdam) 20, 325 (2004).

${ }^{12}$ M. Csontos, T. Wojtowicz, X. Liu, M. Dobrowolska, B. Jankó, J. K. Furdyna, and G. Mihály, Phys. Rev. Lett. 95, 227203 (2005).

${ }^{13}$ M. Csontos, G. Mihály, B. Jankó, T. Wojtowicz, X. Liu, and J. K. Furdyna, Nat. Mater. 4, 447 (2005).

${ }^{14}$ K. J. Yee, D. Lee, X. Liu, W. L. Lim, M. Dobrowolska, J. K. Furdyna, Y. S. Lim, K. G. Lee, Y. H. Ahn, and D. S. Kim, J. Appl. Phys. 98, 113509 (2005).

${ }^{15}$ A. V. Kimel, G. V. Astakhov, G. M. Schott, A. Kirilyuk, D. R. Yakovlev, G. Karczewski, W. Ossau, G. Schmidt, L. W. Molenkamp, and Th. Rasing, Phys. Rev. Lett. 92, 237203 (2004). 